\title{
Jacobian, manipulability, condition number and accuracy of parallel robots
}

\author{
J-P. Merlet \\ INRIA, BP 93, 06902 Sophia-Antipolis, France
}

\begin{abstract}
Although the concepts of jacobian matrix, manipulability and condition number have been floating around since the early beginning of robotics their real significance is not always well understood, although these conditioning indices play an important role e.g. for optimal robot design. In this paper we re-visit these concepts for parallel robots and exhibit some surprising results (at least for the author!) that show that these concepts have to be manipulated with care for a proper understanding of the kinematics behavior of a robot.
\end{abstract}

\section{Introduction}

Parallel robots are nowadays leaving academic laboratories and are finding their way in an increasingly larger number of application fields such as telescopes, fine positioning devices, fast packaging, machine-tool, medical application. A key issue for such use is optimal design as performances of parallel robots are very sensitive to their dimensioning. Optimal design methodologies have to rely on kinetostatic performance indices and accuracy is clearly a key-issue for many applications. It has also be a key-issue for serial robots and consequently this problem has been extensively studied and various accuracy indices have been defined. The results have been in general directly transposed to parallel robots. We will now review how well these indices are appropriate for parallel robots.

\section{Jacobian and inverse Jacobian matrix}

Let $\mathbf{X}_{\mathbf{a}}$ denotes the generalized coordinates of the end-effector composed of parameters describing the available $n$ d.o.f. of the end-effector while $\mathbf{X}$ denotes all the generalized coordinates of the end-effector. We will impose no constraints on the choice of $\mathbf{X}$ (e.g. for a Gough robot with a planar platform the pose may be represented by the 9 coordinates of 3 particular points on the end-effector).

The geometry of the robot is described by its joints variables vector $\Theta$. The twist $\mathbf{W}$ of the end effector is composed of its translational and angular velocities and the restricted twist $\mathbf{W}_{\mathbf{a}}$ is defined as the restriction of $\mathbf{W}$ to the available d.o.f. of the robot. It is well known that for robot having at least 2 rotational d.o.f. $\mathbf{W}$ is not the time-derivative of $\mathbf{X}$ as there is no representation of the orientation whose derivatives corresponds to the angular velocities. However there exists usually a matrix $\mathrm{H}$ such that

$$
\mathbf{W}=\mathrm{H} \dot{\mathbf{X}}
$$


In the usual approach the jacobian matrix $\mathrm{J}_{\mathrm{k}}$ linearly relates the actuated joint velocities $\dot{\Theta}_{\mathbf{a}}$ to $\mathbf{W}_{\mathbf{a}}$ :

$$
\mathbf{W}_{\mathbf{a}}=\mathrm{J}_{\mathrm{k}} \dot{\Theta}_{\mathbf{a}}
$$

In this paper we consider only non-redundant robots so that matrix $\mathrm{J}_{\mathrm{k}}$ is square and we will call it the kinematic jacobian. A feature of parallel robots is that it is usually easy to establish an analytical form for $\mathrm{J}_{\mathrm{k}}^{-1}$ while it is often impossible to obtain $\mathrm{J}_{\mathrm{k}}$.

But we may also define other jacobian matrices by first changing the parameters in $\boldsymbol{\Theta}$. Indeed parallel robots differ from their serial counterpart by a larger number of passive joints and it may thus be interesting to include the $m$ passive joints variables $\boldsymbol{\Theta}_{p}$. If $\boldsymbol{\Theta}$ is defined as $\left(\boldsymbol{\Theta}_{\boldsymbol{a}}, \boldsymbol{\Theta}_{p}\right)$ we may then define write the $l$ inverse kinematics equations as $\mathbf{F}\left(\boldsymbol{\Theta}, \mathbf{X}_{\mathbf{a}}\right)=0$ from which we derive

$$
\frac{\partial \mathbf{F}}{\partial \boldsymbol{\Theta}} \dot{\boldsymbol{\Theta}}+\frac{\partial \mathbf{F}}{\partial \mathbf{X}_{\mathbf{a}}} \dot{\mathbf{X}}_{\mathbf{a}}=\mathrm{U} \dot{\boldsymbol{\Theta}}+\mathrm{V}_{\mathrm{a}} \dot{\mathbf{X}}_{\mathbf{a}}=0
$$

where $\mathrm{U}$ is $(l \times(n+m))$ and $\mathrm{V}_{\mathrm{a}}$ is $(l \times n)$. This relation allows to quantify the influence of the measurement errors on the passive and actuated joints variables on the positioning errors $\Delta \mathbf{X}_{\mathbf{a}}$ on the $n$ d.o.f. of the end-effector by using (1).

Although we say that some robot have $n<6$ d.o.f., still the end-effector is a 6 d.o.f. rigid body and positioning errors on all d.o.f. should be examined. It is thus interesting to determine an inverse jacobian that involves the full twist $\mathbf{W}$ of the end-effector. In that case we write the kinematics equations as $\mathbf{G}(\boldsymbol{\Theta}, \mathbf{X})=0$. If we fix $\mathbf{X}$ we know that these kinematics equations have a finite number of solutions, which implies that the number of equations in $\mathbf{G}$ should be $n+m$. By differentiation we get:

$$
\frac{\partial \mathbf{G}}{\partial \boldsymbol{\Theta}} \dot{\boldsymbol{\Theta}}+\frac{\partial \mathbf{G}}{\partial \mathbf{X}} \dot{\mathbf{X}}=\mathrm{A} \dot{\boldsymbol{\Theta}}+\mathrm{B} \dot{\mathbf{X}}=0
$$

where A is a square $n+m \times n+m$ matrix while $\mathrm{B}$ is $n+m \times 6$. Provided that $\mathrm{H}$ is square and not singular we may now derive an inverse jacobian such that

$$
\dot{\Theta}=-\mathrm{A}^{-1} \mathrm{BH}^{-1} \mathbf{W}=\mathrm{J}^{-1} \mathbf{W}
$$

where $\mathrm{J}^{-1}$ is $n+m \times 6$ In most cases however a velocity analysis allows one to obtain a simpler inverse jacobian matrix through a relation that involves only $\dot{\Theta_{a}}$ :

$$
\left(\begin{array}{c}
\dot{\Theta_{a}} \\
\mathbf{O}
\end{array}\right)=J_{\mathrm{fk}}^{-1} \mathbf{W}
$$

where $\mathrm{J}_{\mathrm{fk}}^{-1}$ is $n+m \times 6$ and will be called the full inverse kinematics jacobian.

We may further extend this approach to take into account the design parameters $\mathcal{P}$ of the robot (e.g. the location of the anchor points of the legs in a Gough platform). For that purpose the kinematics equations will be written as $\mathbf{G}(\mathcal{P}, \boldsymbol{\Theta}, \mathbf{X})=0$ and the matrix of the partial derivatives of $\mathbf{G}$ with respect to $\mathcal{P}$ will allow one to quantify the influence of the errors on $\mathcal{P}$ on the positioning error of the end-effector. 
As may be seen there is not a single inverse jacobian matrix but a multiplicity of them. Note however an important property of the inverse jacobian $\mathrm{J}^{-1}$ of (5) with respect to $\mathrm{J}_{\mathrm{fk}}^{-1}$ : the rank of $\mathrm{J}_{\mathrm{fk}}^{-1}$ is the same than the rank of $\mathrm{J}^{-1}$.

It is also important to note that any inverse jacobian involving the full twist of the end-effector $\mathbf{W}$ will not be homogeneous in terms of units. This will be true also for the inverse kinematic jacobian for robot involving both translation and rotational d.o.f. for the end-effector. Consequently many matrix properties (such as the trace, determinant) will not be invariant under a change of units.

In this paper we will focus on the influence of $\Delta \Theta_{a}$ on the positioning errors of the end-effector through $\mathrm{J}_{\mathrm{fk}}^{-1}$. The necessity of using the full inverse kinematic jacobian will be emphasized on an example.

\subsection{Example: the $3-U \underline{P} U$ robot}

Tsai [10] has proposed this robot as a 3 d.o.f. translation robot (figure 1). Each leg of this robot is constituted, starting from the base, by a U joint followed by an extensible leg terminated by another $\mathrm{U}$ joint whose axis are the same than the $U$ joint on the base. This constraint allows theoretically to obtain only translation for the end-effector. This example will allow

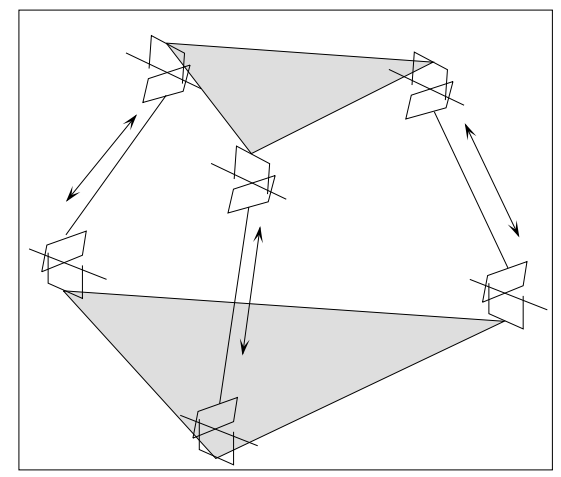

Figure 1: The $3-U \underline{P} U$ robot

us to establish a methodology for determining the full inverse kinematic jacobian. But it will also enable to show the importance of this matrix. The story is that such a robot was designed at Seoul National University (SNU) and that is was exhibiting a strange behavior: although the prismatic actuators were locked, the end-effector was exhibiting significant orientation motion. This phenomena was explained by Bonev and Zlatanov [1] and later in $[2,11]$. Furthermore motion sensitivity to manufacturing tolerances has been studied $[5,8]$ and has shown that this robot was very sensitive. 
We will denote by $B_{1}, B_{2}, B_{3}$ the center of the $\mathrm{U}$ joints on the platform and will now calculate the full inverse kinematic jacobian matrix. The velocity $\mathbf{V}_{\mathbf{B}}$ of the $B$ points is $\mathbf{V}_{\mathbf{B}}=\mathbf{V}+\mathbf{B} \mathbf{C} \times \mathbf{\Omega}$. Let us define $\mathbf{n}$ as the unit vector of the leg and compute the dot product of the right and left terms of the previous equation:

$$
\mathbf{V}_{\mathbf{B}} \cdot \mathbf{n}=\dot{\rho} \mathbf{n}=\mathbf{V} \cdot \mathbf{n}+(\mathbf{B C} \times \boldsymbol{\Omega}) \cdot \mathbf{n}=\mathbf{V} \cdot \mathbf{n}+(\mathbf{C B} \times \mathbf{n}) \cdot \boldsymbol{\Omega}
$$

Now let us define $\mathbf{u}_{\mathbf{i}}, \mathbf{v}_{\mathbf{i}}$ the unit vectors of the two joint axis of the $\mathrm{U}$ joint at $B_{i}$. These vectors are the same for the base and platform. The angular velocity of the leg $\boldsymbol{\omega}_{l}$ with respect to the base and the angular velocity of the platform $\omega_{p}$ with respect to the leg are

$$
\boldsymbol{\omega}_{\boldsymbol{l}}=\dot{\theta}_{A}^{i} \mathbf{u}_{\mathbf{i}}+\dot{\alpha}_{A}^{i} \mathbf{v}_{\mathbf{i}} \quad \boldsymbol{\omega}_{\boldsymbol{p}}=\dot{\theta}_{B}^{i} \mathbf{u}_{\mathbf{i}}+\dot{\alpha}_{B}^{i} \mathbf{v}_{\mathbf{i}}
$$

The angular velocity of the platform is

$$
\boldsymbol{\Omega}=\omega_{l}+\omega_{p}=K_{1}^{i} \mathbf{u}_{\mathbf{i}}+K_{2}^{i} \mathbf{v}_{\mathbf{i}}
$$

where $K_{1}^{i}, K_{2}^{i}$ can be obtained from the previous equations. Now define $\mathbf{s}_{i}=\mathbf{u}_{\mathbf{i}} \times \mathbf{v}_{\mathbf{i}}$ and compute the dot product of the right and left terms of the previous equation by $\mathbf{s}_{\mathbf{i}}$ :

$$
\mathrm{s}_{\mathbf{i}} \cdot \Omega=0
$$

Combining equations $(7,8)$ we get the full velocities equations involving the twist $\mathbf{W}$ as

$$
\left(\begin{array}{c}
\dot{\rho}_{i} \\
\mathbf{0}
\end{array}\right)=\mathrm{J}_{\mathrm{fk}}^{-1} \mathbf{W}=\left(\begin{array}{ll}
\mathbf{n}_{\mathbf{i}} & \left(\mathbf{C B}_{\mathbf{i}} \times \mathbf{n}_{\mathbf{i}}\right) \\
\mathbf{0} & \mathbf{s}_{\mathbf{i}}
\end{array}\right) \mathbf{W}
$$

which establish the full inverse kinematic jacobian. The inverse kinematic jacobian may be extracted from $\mathrm{J}_{\mathrm{fk}}^{-1}$ as the $3 \times 3$ matrix whose rows are the $\mathbf{n}_{\mathbf{i}}$ vectors. But an important point for accuracy analysis is to consider the lower part of $\mathrm{J}_{\mathrm{fk}}^{-1}$ which shows that if $\mathbf{s}_{\mathbf{1}} \cdot\left(\mathbf{s}_{\mathbf{2}} \times \mathbf{s}_{\mathbf{3}}\right)=0$ the platform may exhibit orientation motion that may be infinitesimal or finite according to the geometry of the $\mathrm{U}$ joint. It happens that the design of the SNU robot was in the later category.

\section{Manipulability}

It is realistic to assume that the joint errors are bounded and consequently so will be the positioning errors. The norm of the bound may be chosen arbitrary as (6) is linear so that a simple scaling will allow to determine the positioning error from the errors obtained for a given bound. A value of 1 for the bound is usually chosen:

$$
\|\Delta \Theta\| \leq 1
$$




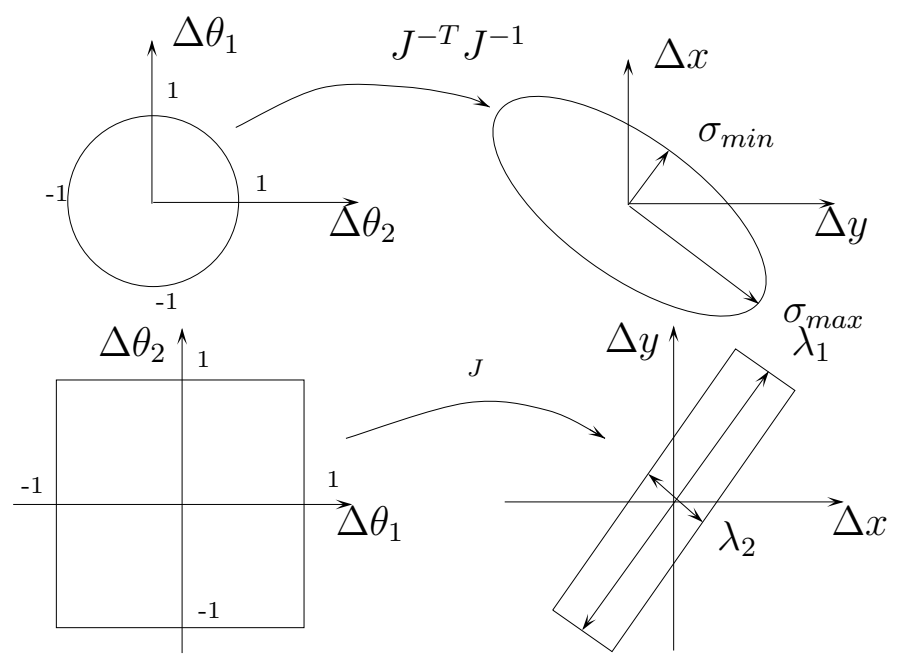

Figure 2: The mapping between the joints errors space and the generalized coordinates error space induced by $\mathrm{J}^{-\mathrm{T}} \mathrm{J}$ according to the norm: on top the Euclidean norm and on bottom the infinity norm.

which leads to

$$
\boldsymbol{\Delta} \mathbf{X}^{T} \mathrm{~J}^{-\mathrm{T}} \mathrm{J}^{-1} \boldsymbol{\Delta} \mathrm{X} \leq 1
$$

A classical geometrical interpretation of this relation is presented for the $2 \mathrm{D}$ case in figure 2. If the Euclidean norm is used (10) represents a circle in the joints errors space. This circle is mapped through matrix $\mathrm{J}^{-\mathrm{T}} \mathrm{J}^{-1}$ into an ellipse in the generalized coordinates error space. More generally the mapping transform the hyper-sphere of the joints errors space into an ellipsoid, usually called the manipulability ellipsoid.

In fact the use of the Euclidean norm is not realistic: it implies for example that if one of the joint error is 1 , then by some mysterious influence all the other joint errors are 0 . The appropriate norm is the infinity norm that states that the absolute value of the joint errors are independently bounded by 1 . With this norm (10) represents a $n$-dimensional square in the joints errors space that is mapped into the kinematics polyhedron, that includes the manipulability ellipsoid, in the generalized coordinates errors space. Figure 2 illustrates this mapping in the $2 \mathrm{D}$ case. It must be noted that, apart of being more realistic, the previous mapping leads to geometrical object that can be more easily manipulated than the ellipsoid. For example assume that one want to determine what are all the possible end-effector velocities that can be obtained in 2 different poses of the end-effector. For that purpose we will have to calculate the intersection of the 2 polyhedra obtained for the 2 poses, a well known problem of computational geometry, that can be much more easily solved than computing the intersection of 2 
ellipsoids.

\section{Condition number}

A large dimension along a given axis of the kinematics polyhedron indicates a large amplification error. It is therefore necessary to quantify this amplification factor. Let us consider the linear system:

$$
\mathrm{J}^{-1} \Delta \mathrm{X}=\Delta \Theta,
$$

where $\mathrm{J}^{-1}$ is a $n \times n$ inverse kinematic jacobian matrix. The error amplification factor in this system expresses how a relative error in $\Theta$ gets multiplied and leads to a relative error in $\mathbf{X}$. It characterize in some sense the dexterity of the robot and will be used as a performance index. We now use a norm such that

$$
\left\|\mathrm{J}^{-1} \Delta \mathbf{X}\right\| \leq\left\|\mathrm{J}^{-1} \mid\right\|\|\Delta \mathbf{X}\|
$$

and obtain

$$
\frac{\|\Delta \mathbf{X}\|}{\|\mathbf{X}\|} \leq\left\|J^{-1}\right\|\|||\| \frac{\|\Delta \boldsymbol{\Theta}\|}{\|\Theta\|}
$$

The error amplification factor, called the condition number $\kappa$, is therefore defined as

$$
\kappa\left(\mathrm{J}^{-1}\right)=\left\|\mathrm{J}^{-1}|||| \mathrm{J}\right\|
$$

The condition number is thus dependent on the choice of the matrix norm. The most used norms are:

- the 2-norm defined as the square root of the largest eigenvalue of matrix $\mathrm{J}^{-\mathrm{T}} \mathrm{J}^{-1}$ : the condition number of $\mathrm{J}^{-1}$ is thus the square root of the ratio between the largest and the smallest eigenvalues of $\mathrm{J}^{-\mathrm{T}} \mathrm{J}^{-1}$,

- the Euclidean (or Frobenius) norm defined for the $m \times n$ matrix A by: $\|\mathrm{A}\|=\sqrt{\sum_{i=1}^{i=m} \sum_{j=1}^{j=n}\left|a_{i j}\right|^{2}}$ or equivalently as $\sqrt{\operatorname{tr}\left(\mathrm{A}^{\mathrm{T}} \mathrm{A}\right)}$ : if $\lambda_{i}$ denotes the eigenvalues of $\mathrm{J}^{-\mathrm{T}} \mathrm{J}^{-1}$, then the condition number is the ratio between $\sum \lambda_{i}^{2}$ and $\prod \lambda_{i}$. Note that sometime is also used a weighted Frobenius norm in which $\mathrm{A}^{\mathrm{T}} \mathrm{A}$ is substituted by $\mathrm{A}^{\mathrm{T}} \mathrm{WA}$ where $\mathrm{W}$ is the weight matrix

In these two cases, the smallest possible value of the condition number is 1 . The inverse of the condition number, which has a value in $[0,1]$, is also often used. A value of 0 will indicate that the inverse jacobian matrix is singular.

The condition number is quite often used as an index to describe first the accuracy/dexterity of a robot and, second, the closeness of a pose to a singularity. For the later point it is in general not possible to define a mathematical distance to a singularity for robots whose d.o.f. is a mix between translation and orientation: hence the use of the condition number is as valid an index than any other one. But it has the advantage of being a single number for describing the overall kinematic behavior of a robot. 
The definition of the condition number makes clear that we cannot calculate its analytical form as a function of the pose parameters except for very simple robot. But robust linear algebra software allows to calculate it numerically for a given pose.

But for robot having both translation and orientation d.o.f. there is a major drawback of the condition number: the matrix involved in its calculation are not homogeneous in terms of units. Hence the value of the condition number for a given robot and pose will change according to the unit choice, while clearly the kinematic accuracy is constant. Ma and Angeles [6] suggested to define a normalized inverse jacobian matrix by dividing the rotational elements of the matrix by a length such as the length of the links in a nominal position, or the natural length defined as that which minimizes the condition number for a given pose. Still the choice of the length remains arbitrary as it just allows to define a correspondence between a rotation and a translation and as mentioned by Park [9] "this arbitrariness is an unavoidable consequence of the geometry of $\mathrm{SE}(3)$ ".

To evaluate the efficiency of the condition number for accuracy evaluation we just use our Gough robot and chooses three reference poses defined by the coordinates of the center and the Euler angles as $P_{1}=x=y=0, z=53$ cm, $\psi=0, \theta=0, \phi=0$ (roughly the pose obtained for the mid-stroke of the actuator), $P_{2}=x=y=0, z=53 \mathrm{~cm}, \psi=30^{\circ}, \theta=0, \phi=0$ (whose orientation is roughly $1 / 3$ of the possible rotation around the $z$ axis) and $P_{3}=x=y=10, z=53 \mathrm{~cm}, \psi=0, \theta=0, \phi=0$.(close to the border of the translation workspace for this orientation). We then computed the absolute value of the maximal positioning error at these poses, obtained as the sum of the absolute value of the elements of the rows of the kinematic jacobian, as indicated in the following table.

\begin{tabular}{|l|l|l|l|l|l|l|}
\hline Pose & $\Delta X_{x}$ & $\Delta X_{y}$ & $\Delta X_{z}$ & $\Delta X_{\theta_{x}}$ & $\Delta X_{\theta_{y}}$ & $\Delta X_{\theta_{z}}$ \\
\hline$P_{1}$ & 0.1184 & 0.1268 & 0.010087 & 0.1185 & 0.1184 & 0.697 \\
$P_{2}$ & 0.1189 & 0.1274 & 0.01266 & 0.1333 & 0.1429 & 0.808 \\
$P_{3}$ & 0.123 & 0.1309 & 0.0372 & 0.15 & 0.1663 & 0.7208 \\
\hline
\end{tabular}

It can be seen in this table that the positioning errors are significantly larger for $P_{2}$ and $P_{3}$ compared to $P_{1}$. As for $P_{3}$ the errors are usually larger compared to $P_{2}$ except for the rotation around $z$. Hence as far as accuracy is concerned the ordering of the poses from the most to the least accurate is $P_{1}, P_{2}, P_{3}$ and we expect to obtain a similar ordering for the condition number.

For this robot we define the normalized inverse jacobian matrix $\mathrm{J}_{\mathrm{n}}^{-1}$ obtained by dividing the orientation components of the $\mathrm{J}_{\mathrm{k}}^{-1}$ by 53 i.e. roughly the legs lengths at pose $P_{1}$. The considered accuracy indices will be

- $C_{d}$ : the determinant of $\mathrm{J}_{\mathrm{k}}^{-1}$

- $C_{2}, C_{2}^{n}$ : the 2-norm condition number of $\mathrm{J}_{\mathrm{k}}^{-1}, \mathrm{~J}_{\mathrm{n}}^{-1}$

- $C_{F}, C_{F}^{n}$ : the Frobenius-norm condition number of $\mathrm{J}_{\mathrm{k}}^{-1}, \mathrm{~J}_{\mathrm{n}}^{-1}$ 
- $C_{2}^{3}, C_{F}^{3}$ : the 2-norm and Frobenius norm condition number of the inverse jacobian matrix obtained when the inverse kinematics equations are based on the coordinates of 3 points of the end-effector. The chosen points will be all possible triplets in the set $B_{i}$ : hence we will provide ranges for these indices.

The results are presented in the following table:

\begin{tabular}{|l|l|l|l|l|l|l|l|}
\hline & $C_{d}$ & $C_{2}$ & $C_{2}^{n}$ & $C_{F}$ & $C_{F}^{n}$ & $C_{2}^{3}$ & $C_{F}^{3}$ \\
\hline$P_{1}$ & -29.22 & 75.14 & 63.9 & 152.8 & 70.2 & {$[9.55,55.47]$} & {$[258.8,3204.9]$} \\
$P_{2}$ & -24.64 & 75.16 & 73.8 & 154 & 80.9 & {$[9.62,43.84]$} & {$[218.8,2383.6]$} \\
$P_{3}$ & -23.93 & 80.65 & 68.4 & 158.3 & 74.7 & {$[10.06,58.95]$} & {$[286.5,3618]$} \\
\hline
\end{tabular}

For $C_{2}$ it may be seen that the difference is surprisingly very small between $P_{1}, P_{2}$ and significant between $P_{3}, P_{2}$. The ordering between $P_{2}, P_{3}$ is not respected for $C_{2}^{n}, C_{F}^{n}$ although these indices are coherent when considering $P_{1}$. For $C_{F}, C_{d}$ the ordering is respected although the changes in the index are relatively small for $C_{F}$. On the other hand there is a surprisingly decrease of $C_{2}^{3}, C_{F}^{3}$ between $P_{2}$ and $P_{1}$ while there is a significant increase between $P_{1}$ and $P_{3}$. Hence none of this condition numbers exhibits a completely coherent behavior with respect to the accuracy of this robot.

This simple example shows clearly that the concept of condition number has to be carefully considered when talking about optimal design for robot.

\section{Isotropy}

An isotropic pose of a robot is defined as a pose where $\kappa$ is equal to 1 and a robot which has only isotropic poses in its workspace is coined an isotropic robot. Designing an isotropic parallel robot is often considered as a design objective [3, 12]. A trivial example of isotropic robot is a serial Cartesian $\mathrm{X}-\mathrm{Y}-\mathrm{Z}$ robot whose kinematic jacobian matrix is the identity. But this is a surprising denomination as stricto sensu isotropy indicates that the performances of a robot should be the same whatever is the motion direction. Now if we assume that all the actuator velocities of a X-Y-Z robot are bounded to 1 , then the maximal velocity of the end-effector lie in the range $[1, \sqrt{3}]$ : as far as velocity is considered such robot is far from isotropy. Still the concept may have some interest: for example any Cartesian robot whose actuator axis are not mutually orthogonal will exhibit a ratio between its maximal velocities over its workspace that will be larger than $\sqrt{3}$. Hence, instead of using the name "isotropic robot" we may consider using the name "maximally regular robot".

\section{Global conditioning index}

The condition number is a local indication for the dexterity of a robot. To evaluate the dexterity of a robot over a given workspace $W$ Gosselin [4] has 
introduced the global conditioning index (GCI) as:

$$
\mathrm{GCI}=\frac{\int_{W}\left(\frac{1}{\kappa}\right) d W}{\int_{W} d W}
$$

which correspond to the average value of $1 / \kappa$. Clearly this concept makes sense for the optimal design of robot for which the extremal and average value of any performance are important design factors. But apart of the validity of the condition number that has been discussed in a previous section the problem with the GCI is its calculation. Clearly we cannot expect to obtain its closed-form and we must rely on a numerical evaluation. The usual method is to sample the workspace using a regular grid, compute $1 / \kappa_{i}$ at each node $N_{i}$ and approximate the GCI as $\mathrm{GCI}_{\mathrm{a}}$, the sum of the $1 / \kappa_{i}$ divided by the number of nodes. This calculation may be computer intensive as its complexity is exponential with respect to the number of d.o.f. of the robot. Furthermore this method does not allow to get a bound on $\left|\mathrm{GCI}-\mathrm{GCI}_{\mathrm{a}}\right|$. To deal with this error problem it is sometimes assumed that if the result with $m_{1}$ sampling points is close to the result obtained with $m_{2}$ points, $m_{2}$ being significantly larger than $m_{1}$, then the later result is a good approximation of the index. This assumption will be true only if the condition number is smooth enough, a claim that is difficult to support. Consider for example a simple planar serial 2R robot: its GCI can be computed almost exactly as it depends only on a single parameter. We sample this parameter using $10,20, \ldots, m_{1}, m_{2}=m_{1}+10$ points and stop the calculation when the relative error between $\mathrm{GCI}_{\mathrm{a}}\left(m_{1}\right), \mathrm{GCI}_{\mathrm{a}}\left(m_{2}\right)$ is lower than $0.5 \%$ and assumes $\mathrm{GCI} \approx \mathrm{GCI}_{\mathrm{a}}\left(m_{2}\right)$. For $m_{1}=50$ the relative error is $0.377 \%$ while the relative error on the GCI is still $1.751 \%$. It may be assumed that such error will even be larger for more complex robot.

A better evaluation will probably be obtained by using Monte-Carlo integration (with an error that decreases as $1 / \sqrt{n}$ where $n$ is the number of sampling nodes) or quasi-Monte Carlo. In the previous example (which is not favorable for Monte-Carlo method as there is only one parameter) we found out that by using the same stop criteria the relative error on the GCI was reduced to $0.63 \%$. A certified evaluation of the global conditioning index is therefore an open problem but nevertheless the calculation of such index will probably be computer intensive.

\section{Conclusion}

Classical dexterity indices such as the condition number are not very adequate for parallel robots. In our opinion the most appropriate accuracy indices are the determination of the maximal positioning errors, their average values and their variance. We have presented in a recent paper a a computer intensive method for finding the largest maximal positioning errors, up to an arbitrary accuracy, of a 6 d.o.f. robot [7]. A real challenge is to design algorithms for calculating the average and variance of the maximal positioning 
errors over a given workspace. An important point is that there is no need to calculate these values exactly as soon as it is possible to impose a bound on the calculation error. Indeed for comparison purposes an approximate value with a guaranteed error will be sufficient.

\section{References}

[1] Bonev I.A. and Zlatanov D. The mystery of the singular SNU translational parallel robot. www.parallemic.org/Reviews/Review004.html, June, 12, 2001.

[2] Di Gregorio R. and Parenti-Castelli V. Mobility analysis of the 3-UPU parallel mechanism assembled for a pure translational motion. ASME J. of Mechanical Design, 124(2):259-264, June 2002.

[3] Fattah A. and Hasan Ghasemi A.M. Isotropic design of spatial parallel manipulators. Int. J. of Robotics Research, 21(9):811-824, September 2002.

[4] Gosselin C. Kinematic analysis optimization and programming of parallel robotic manipulators. Ph.D. Thesis, McGill University, Montréal, June, 15, 1988.

[5] Han C. and others . Kinematic sensitivity analysis of the 3-UPU parallel manipulator. Mechanism and Machine Theory, 37(8):787-798, 2002.

[6] Ma O. and Angeles J. Optimum architecture design of platform manipulator. In $I C A R$, pages 1131-1135, Pise, June, 19-22, 1991.

[7] Merlet J-P. and Daney D. Dimensional synthesis of parallel robots with a guaranteed given accuracy over a specific workspace. In IEEE Int. Conf. on Robotics and Automation, Barcelona, April, 19-22, 2005.

[8] Parenti-Castelli V. and Di Gregorio R. Influence of manufacturing errors on the kinematic performance of the 3-UPU parallel mechanism. In 2nd Chemnitzer Parallelkinematik Seminar, pages 85-99, Chemnitz, April, 12-13, 2000.

[9] Park M.K. and Kim J.W. Kinematic manipulability of closed chains. In $A R K$, pages 99-108, Portoroz-Bernadin, June, 22-26, 1996.

[10] Tsai L-W. Kinematics of a three-dof platform with three extensible limbs. In $A R K$, pages 401-410, Portoroz-Bernadin, June, 22-26, 1996.

[11] Wolf A., Shoham M., and Park F.C. Investigation of singularities and self-motions of the 3-UPU robot. In $A R K$, pages 165-174, Caldes de Malavalla, June 29- July 2, 2002.

[12] Zanganeh K.E. and Angeles J. On the isotropic design of general sixdegree-of-freedom parallel manipulators. In J-P. Merlet B. Ravani, editor, Computational Kinematics, pages 213-220. Kluwer, 1995. 